\title{
The role of intravascular ultrasound scan and thin-sliced coronary computed tomography angiography in diagnosing aortic dissection causing acute myocardial infarction
}

\author{
Daisuke Nagatomo, Daizaburo Yanagi, Takeshi Serikawa, \\ Masanori Okabe, Yusuke Yamamoto
}

\section{ABSTRACT}

Introduction: Acute aortic dissection is a disease of high mortality. The symptoms may mimic other conditions and misdiagnosed, such as acute coronary syndrome, coronary involvement complicates the clinical scenario and increases mortality. Case Report: We herein report a case of an acute myocardial infarction caused by acute aortic dissection. Without noticing the aortic dissection, we performed emergent coronary angiography, which showed severe stenosis of the proximal right coronary artery. Intravascular ultrasound scan led us to suspect aortic dissection. However, we performed balloon angioplasty because the patient's hemodynamic status was unstable. ECG-gated coronary computed tomography angiography provided a definitive diagnosis, and the patient underwent successful surgical repair of the aortic dissection. Conclusion:

Daisuke Nagatomo ${ }^{1}$, Daizaburo Yanagi ${ }^{1}$, Takeshi Serikawa², Masanori Okabe ${ }^{3}$, Yusuke Yamamoto ${ }^{4}$

Affiliations: ${ }^{1} \mathrm{MD}$, Resident Physician, Department of Cardiology, Cardiovascular and Aortic Center of Saiseikai Fukuoka General Hospital, Fukuoka, JAPAN; ${ }^{2}$ MD, Manager of the Catheterization Laboratory, Department of Cardiology, Cardiovascular and Aortic Center of Saiseikai Fukuoka General Hospital, Fukuoka, JAPAN; ${ }^{3} \mathrm{MD}$, Assistant Director, Department of Cardiology, Cardiovascular and Aortic Center of Saiseikai Fukuoka General Hospital, Fukuoka, JAPAN; ${ }^{4} \mathrm{MD}$, Director, Department of Cardiology, Cardiovascular and Aortic Center of Saiseikai Fukuoka General Hospital, Fukuoka, JAPAN.

Corresponding Author: Daisuke Nagatomo, 1-3-46 Tenjin, chuo-ku, Fukuoka-shi, Fukuoka, JAPAN.810-0001; Tel: +8192-771-8151, Fax: +81-92-716-0185; Email: miserybeatle@ me.com

Received: 19 October 2013

Accepted: 23 November 2013

Published: 01 April 2014
Acute coronary syndrome associated with acute aortic dissection is not rare. However, the management of these conditions depends on the details of each case. This case demonstrates the difficulty of treating such cases in the real world. Herein, we describe educational imaging findings and briefly discuss the management of cases involving acute coronary syndrome associated with acute aortic dissection.

Keywords: Coronary computed tomography angiography, Acute aortic dissection, Acute myocardial infarction

\section{How to cite this article}

Nagatomo D, Yanagi D, Serikawa T, Okabe M, Yamamoto Y. The role of intravascular ultrasound scan and thin-sliced coronary computed tomography angiography in diagnosing aortic dissection causing acute myocardial infarction. International Journal of Case Reports and Images 2014;5(4):302-306.

doi:10.5348/ijcri-201462-CR-10373

\section{INTRODUCTION}

Patients with acute aortic dissection (AAD) mayinitially present with only signs of acute coronary syndrome (ACS), such as ST elevation on electrocardiograms (ECGs). In such situations, the correct diagnosis may be missed. A diagnosis of acute coronary syndrome may lead to the inappropriate administration of thrombolytic agents, resulting in catastrophic consequences. Transthoracic echocardiography is useful as a simple imaging test. However, its diagnostic capability is sometimes insufficient in the emergency room. Although the exact diagnosis can be reached, the management of these conditions remains controversial, with only a few reports in literature. 


\section{CASE REPORT}

A 62-year-old male with the sudden onset severe chest pain was transferred to our emergency room by ambulance. He had a history of aortic valve replacement (AVR) due to aortic regurgitation of the tricuspid valve four years earlier. The AVR had thus been performed using a mechanical valve and the prothrombin timeinternational normalized level at admission was 1.76 . He did not suffer from back pain and no laterality of the blood pressure was observed. An ECG showed ST-segment elevation in leads II, III and aVF (Figure 1), suggesting inferior acute myocardial infarction (AMI). A chest X-ray showed no abnormalities. On the trans thoracic echocardiography neither mechanical valve failure or cardiac tamponade was observed. We performed emergent coronary angiography, which revealed a long tight lesion in the proximal segment of the right coronary artery (RCA) (Figure 2) and normal left coronary artery. We planned to perform emergent percutaneous coronary intervention (PCI), however, intravascular ultrasound (IVUS) scan performed before PCI revealed a hypoechoic mass around the stenoticlesion. The narrowing lumen appeared not to be occupied by thrombi, but rather was oppressed by the surrounding mass (Figure 3). These findings suggested that ascending aortic dissection had caused AMI. Although we considered emergent surgical repair, we decided to perform PCI first because the ST level was still elevated and the patient's hemodynamic status was unstable. Balloon angioplasty improved the flow of the RCA, and the hemodynamics was stabilized. We did

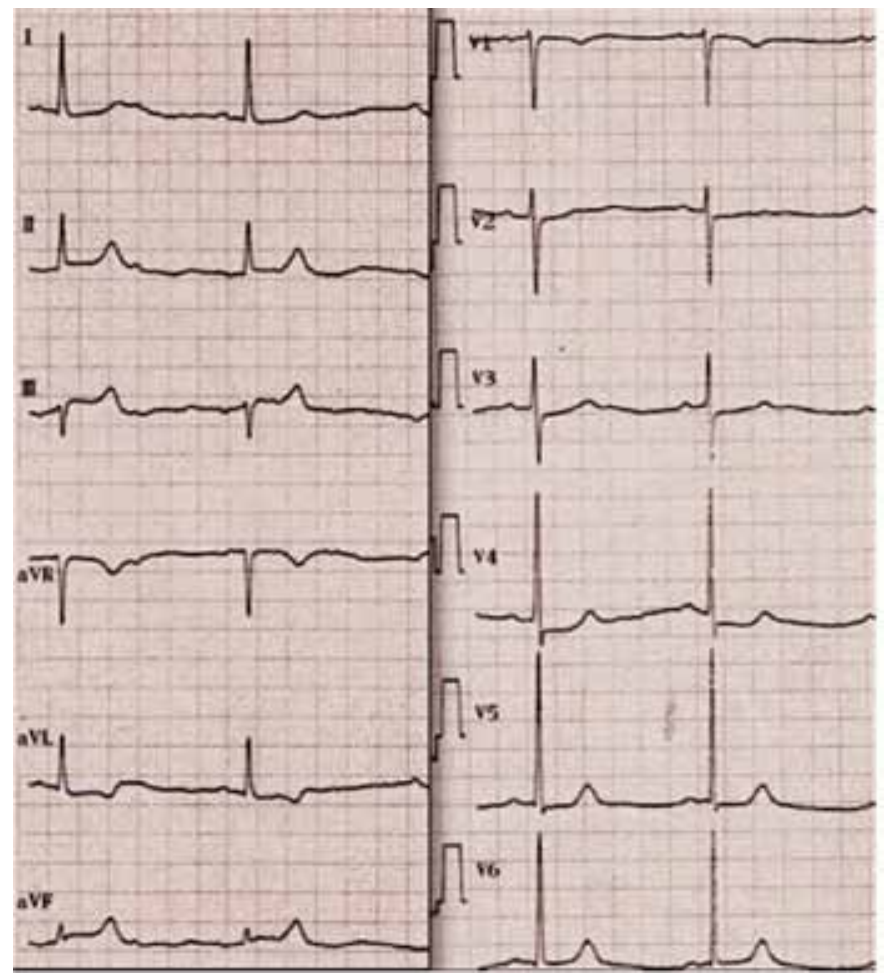

Figure 1: An electrocardiograms showing ST-segment elevation in leads II, III and aVF, suggesting acute myocardial infarction.

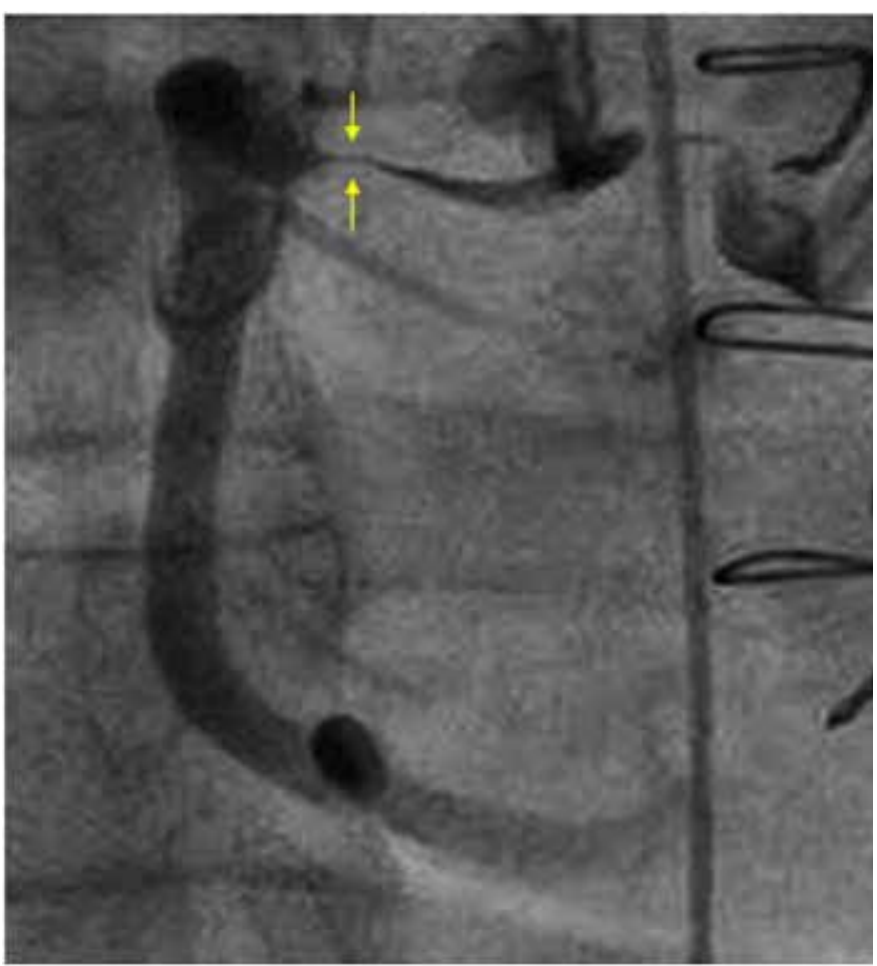

Figure 2: Coronary angiography showing a long, tight lesion in the proximal right coronary artery (arrow).

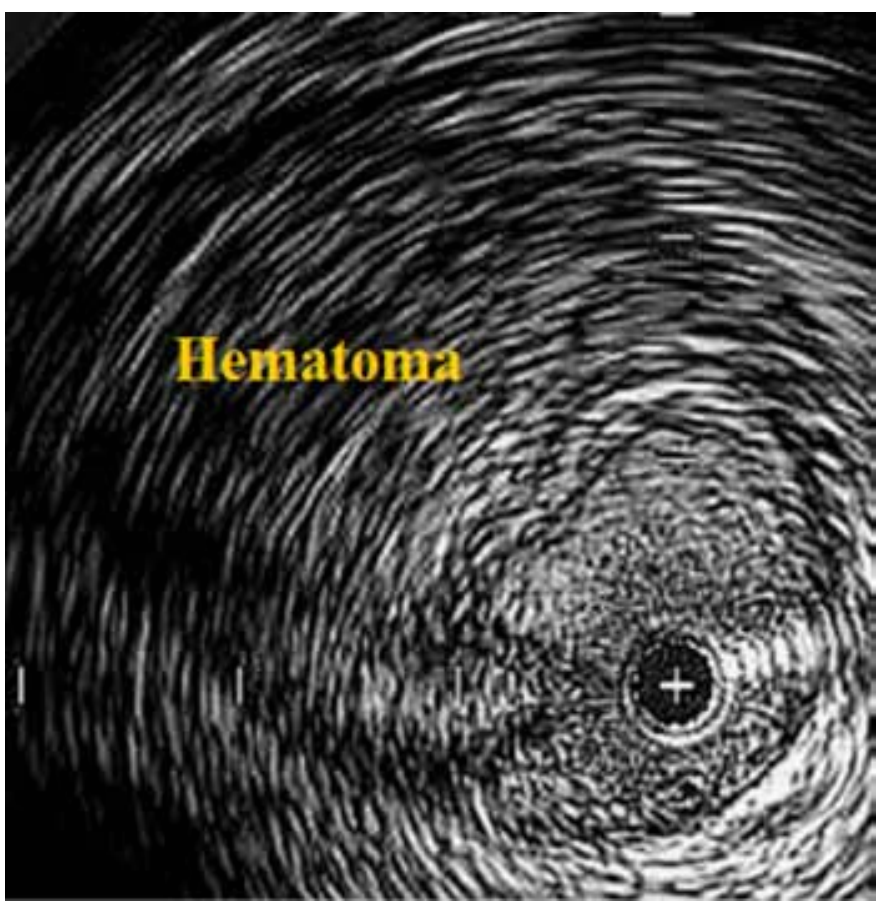

Figure 3: On intravascular ultrasound scan, a hematoma (arrow) was observed around the coronary artery. The narrowing lumen of the right coronary artery did not appear to be occupied by atheromatous plaque or thrombi but rather oppressed by the surrounding mass. The mass continued to the aorta on manual pull-back of the intravascular ultrasound catheter. 
not implant any stents because we wished to avoid the use of antiplatelet agents and did not recognize the acute recoil after balloon angioplasty. Acute aortic dissection was definitely diagnosed following contrast-enhanced computed tomography (CT) angiography (Figures 4 and 5), which clearly showed that the proximal RCA was embedded and oppressed by the intramural hematoma (Figure 5A). The left main trunk was mildly oppressed by the communicating false lumen of the dissection (Figure $\left.{ }_{5} \mathrm{~B}-\mathrm{C}\right)$. The patient underwent successful surgical repair of the aortic dissection.

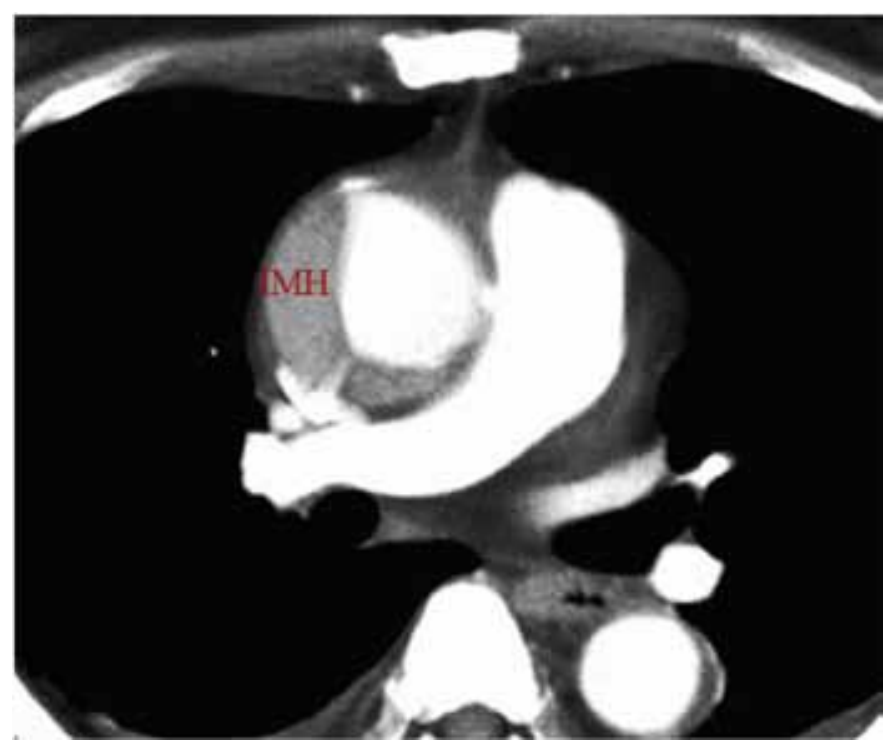

Figure 4: Computed tomography scan showing an intramural hematoma in the ascending aorta.

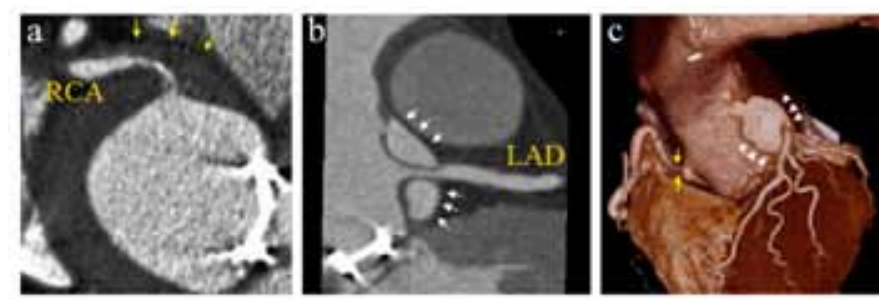

Figure 5: (A) Coronary computed tomography angiography showing the right coronary artery was embedded and oppressed by the intramural hematoma (yellow arrow), (B) On multiplanar reconstruction imaging, the left main trunk appeared to be mildly oppressed by the communicating false lumen (white arrow), (C) Volume rendering imaging showed that the proximal segment of the right coronary artery was oppressed (yellow arrow) and the left main trunk was embedded in the communicating false lumen (white arrowhead).

\section{DISCUSSION}

Acute aortic dissection can occur as one of the most serious complication late complications after AVR. Predictors of AAD after AVR include fragility and thinning of the ascending aorta, aortic dilatation, AR at initial AVR (especially, bicuspid aortic valve) and hypertension [1].
In addition, coronary involvement is a fatal complication of $\mathrm{AAD}$, with a reported incidence of from one to two percent [2]. However, AAD itself sometimes fails to demonstrate any of the classical physical findings, such as a widened mediastinum, aortic regurgitation or the laterality of blood pressure, and up to $30 \%$ of patients suffering from AAD are therefore initially suspected to have other conditions $[3,4]$.

In this case, we could not diagnose $\mathrm{AAD}$ based on either the physical findings, chest X-ray or transthoracic echocardiography in the emergency room even though the patient had a history of AVR. Therefore, if when treating AMI patients in the emergency room, especially those with inferior AMI, clinicians should suspect the existence of aortic dissection at the back of the AMI [4]. However, if aortic dissection cannot be diagnosed in the emergency room in such cases, emergent CAG should be performed. Once AAD is identified as the cause of AMI, the question arised as to how the patient should be managed in the catheterization laboratory? It is controversial to first perform emergent surgical repair of the aorta, or primary PCI before surgery. Furthermore, whether to implant a stentis a difficult choice. The use of strong antiplatelet therapy can result in surgical difficulties, while the strong radial force of the implanted stent would assure a more stable coronary flow. Therefore, this decision should be made based on whether the patient is stable hemodynamically [5]. The findings of IVUS scan and coronary CT angiography in the present case are very educational, as they clearly showed AAD involving the coronary artery. In addition, this case highlights the difficulty of treating similar cases in the real world.

\section{CONCLUSION}

Acute coronary syndrome associated with acute aortic dissection is not rare. However, the management of these conditions depends on the details of each case, and there are many cases that the guidelines cannot be applied. This case demonstrates the difficulty in treating similar cases in the real world. We performed balloon angioplasty, refraining from using stenting before surgery, and subsequently obtained a good result. We believe that this therapeutic regimen is a potential treatment choice in cases involving a poorly disturbed coronary flow.

$$
* * * * * * * * *
$$

\section{Author Contributions}

Daisuke Nagatomo - Conception and design, Acquisition of data, Analysis and interpretation of data, Drafting the article, Critical revision of the article, Final approval of the version to be published

Daizaburo Yanagi - Conception and design, Acquisition of data, Drafting the article, Critical revision of the article, Final approval of the version to be published 
Takeshi Serikawa - Conception and design, Acquisition of data, Drafting the article, Critical revision of the article, Final approval of the version to be published

Masanori Okabe - Conception and design, Critical revision of the article, Final approval of the version to be published

Yusuke Yamamoto - Conception and design, Drafting the article, Critical revision of the article, Final approval of the version to be published

\section{Guarantor}

The corresponding author is the guarantor of submission.

\section{Conflict of Interest}

Authors declare no conflict of interest.

\section{Copyright}

(C) Daisuke Nagatomo et al. 2014; This article is distributed under the terms of Creative Commons attribution 3.0 License which permits unrestricted use, distribution and reproduction in any means provided the original authors and original publisher are properly credited. (Please see www.ijcasereportsandimages.com/ copyright-policy.php for more information.)

\section{REFERENCES}

1. Pieters FA, Wlddershoven JW, Gerardy AC, Geskes G, Cheriex EC, Wellens HJ. Risk of aortic dissection after aortic valve replacement. Am J Cardiol 1993;72(14):1043-7.

2. Erbel R, Alfonso F, Boileau C, et al. Diagnosis and management of aortic dissection. Eur Heart J 2001;22(18):1642-81.

3. Spittell PC, Spittell JA Jr, Joyce JW, et al. Clinical features and differential diagnosis of aortic dissection: Experience with 236 cases (1980 through 1990). Mayo Clin Proc 1993;68(7):642-51.

4. Salvatore Lentini, Sossio Perrotta. Aortic dissection with concomitant acute myocardial infarction: From diagnosis to management. J Emerg Trauma Shock 2011;4(2):273-8.

5. Salvatore Lentini, Luigi Specchia, Antonio Cricco, et al. Hybrid management of acute type A aortic dissection presenting as acute coronary syndrome. Int J Cardiol 2013;167(4):e85-7.

\section{ABOUT THE AUTHORS}

Article citation: Nagatomo D, Yanagi D, Serikawa T, Okabe M, Yamamoto Y. The role of intravascular ultrasound scan and thin-sliced coronary computed tomography angiography in diagnosing aortic dissection causing acute myocardial infarction. International Journal of Case Reports and Images 2014;5(4):302-306.
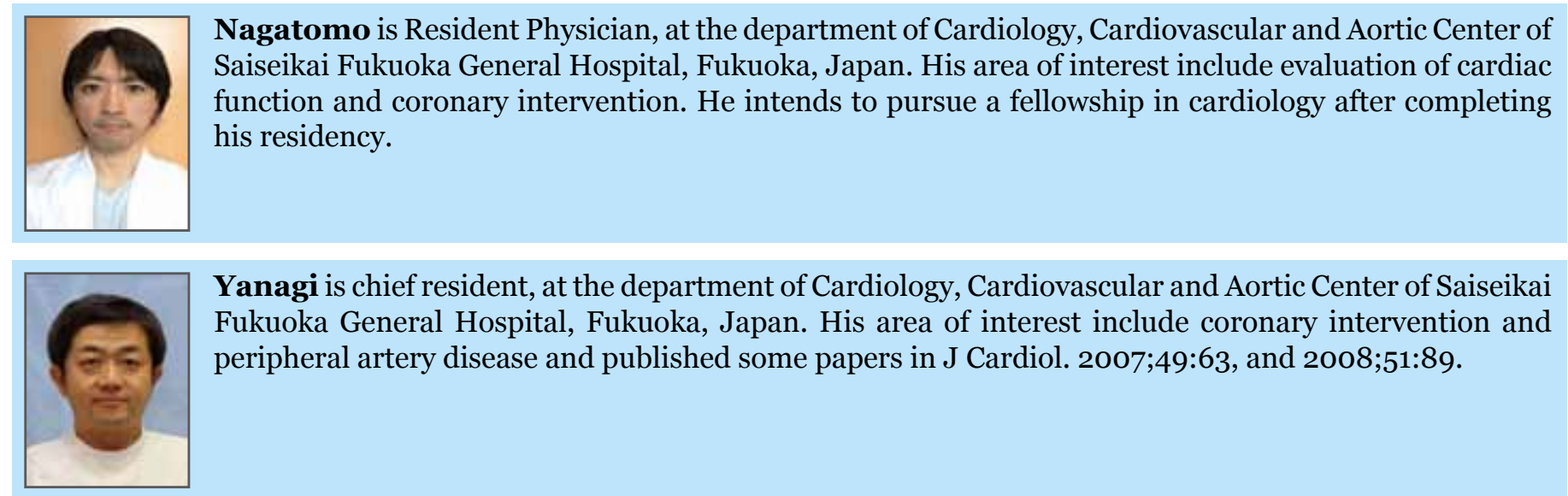

Yanagi is chief resident, at the department of Cardiology, Cardiovascular and Aortic Center of Saiseikai Fukuoka General Hospital, Fukuoka, Japan. His area of interest include coronary intervention and peripheral artery disease and published some papers in J Cardiol. 2007;49:63, and 2008;51:89. 


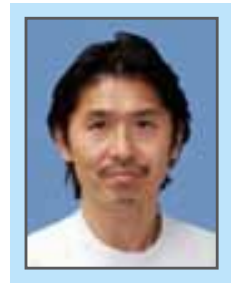

Serikawa is a general manager at the Department of Cardiology Cardiovascular and Aortic Center of Saiseikai Fukuoka General Hospital, Fukuoka Japan. His speciality is coronary intervention(complex PCI and OCT examination). He obtained doctor of Philosophy. He published some papers about coronary intervention and coronary risk factors in J Interv Cardiol. 2011;24:165, and J Cardiol. 2014;63:35.

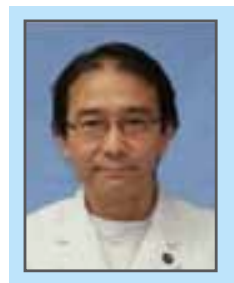

Okabe is Director, at the department of Cardiology, Cardiovascular and Aortic Center of Saiseikai Fukuoka General Hospital, Fukuoka, Japan. His area of interest include myocardial disease and heart failure, and wrote several papers published in Jpn Circ J. 1995;59:49, 1997;61:78, 1999;63:485, Br Heart J. 1991;65:317, Am Heart J. 1992;123:128, Jpn Heart J. 1993;34:121, and Circulation 1997;96:22.

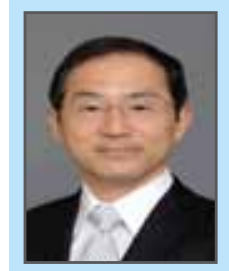

Yamamoto (MD and $\mathrm{PhD}$ ) is now heading Cardiovascular and Aortic Center of Saiseikai Fukuoka General Hospital, Fukuoka, Japan. He is also clinical professor of Kyushu University (Cardiology), Fukuoka, Japan. He researched the pathophysiology of coronary artery spasm at Kyushu University and published several papers in Circulation 1986;74:826, Circulation Research 1987;60:113, 1987;61:772 and JACC 1989;63:33. Then, he studied Cardiac PET in Hammersmith Hospital of London University and wrote some papers in J Nucl Med 1991;32:2169, J Physiol 1992;446:219, Circulation 1992;86:167, 1992;86:1738, 1996;94:792 and 1996;94:808. His present interest is the relationship between ischemic heart disease and environmental issues, especially air pollution.

Access full text article on other devices

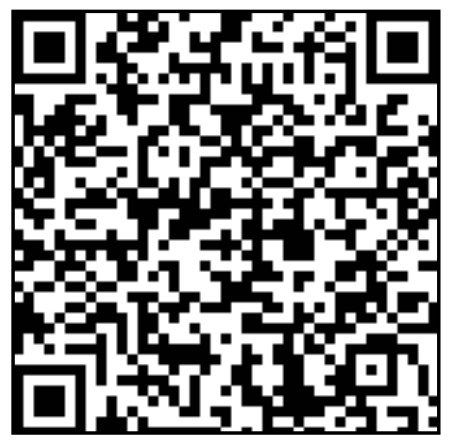

Access PDF of article on other devices

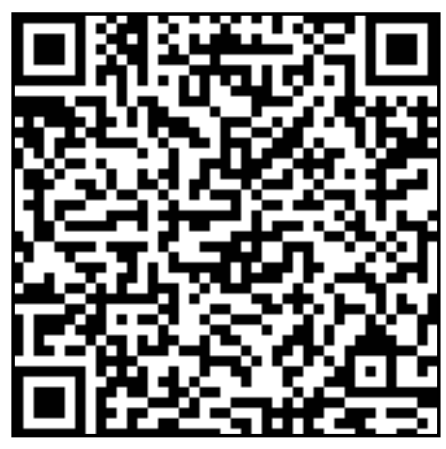

